\title{
Dual-spacecraft reconstruction of a three-dimensional magnetic flux rope at the Earth's magnetopause
}

\author{
H. Hasegawa ${ }^{1}$, B. U. Ö. Sonnerup ${ }^{2}$, S. Eriksson ${ }^{3}$, T. K. M. Nakamura ${ }^{4,}$, and H. Kawano ${ }^{5}$ \\ ${ }^{1}$ Institute of Space and Astronautical Science, Japan Aerospace Exploration Agency, Sagamihara, Japan \\ ${ }^{2}$ Thayer School of Engineering, Dartmouth College, Hanover, New Hampshire, USA \\ ${ }^{3}$ Laboratory for Atmospheric and Space Physics, University of Colorado, Boulder, Colorado, USA \\ ${ }^{4}$ X-Computational Physics Division, Los Alamos National Laboratory, Los Alamos, New Mexico, USA \\ ${ }^{5}$ International Center for Space Weather Science and Education, Kyushu University, Fukuoka, Japan \\ *now at: Space Research Institute, Austrian Academy of Sciences, Graz, Austria \\ Correspondence to: H. Hasegawa (hase@ @stp.isas.jaxa.jp)
}

Received: 21 October 2014 - Revised: 6 January 2015 - Accepted: 9 January 2015 - Published: 3 February 2015

\begin{abstract}
We present the first results of a data analysis method, developed by Sonnerup and Hasegawa (2011), for reconstructing three-dimensional (3-D), magnetohydrostatic structures from data taken as two closely spaced satellites traverse the structures. The method is applied to a magnetic flux transfer event (FTE), which was encountered on 27 June 2007 by at least three (TH-C, TH-D, and TH-E) of the five THEMIS probes near the subsolar magnetopause. The FTE was sandwiched between two oppositely directed reconnection jets under a southward interplanetary magnetic field condition, consistent with its generation by multiple Xline reconnection. The recovered 3-D field indicates that a magnetic flux rope with a diameter of $\sim 3000 \mathrm{~km}$ was embedded in the magnetopause. The FTE flux rope had a significant 3-D structure, because the 3-D field reconstructed from the data from TH-C and TH-D (separated by $\sim 390 \mathrm{~km}$ ) better predicts magnetic field variations actually measured along the TH-E path than does the 2-D Grad-Shafranov reconstruction using the data from TH-C (which was closer to TH-E than TH-D and was at $\sim 1250 \mathrm{~km}$ from TH-E). Such a 3$\mathrm{D}$ nature suggests that the field lines reconnected at the two $\mathrm{X}$-lines on both sides of the flux rope are entangled in a complicated way through their interaction with each other. The generation process of the observed 3-D flux rope is discussed on the basis of the reconstruction results and the pitch-angle distribution of electrons observed in and around the FTE.
\end{abstract}

Keywords. Magnetospheric physics (magnetopause cusp and boundary layers) - space plasma physics (magnetic reconnection; instruments and techniques)

\section{Introduction}

Magnetic field structures and topologies play an important role in dynamical plasma phenomena, such as solar or stellar flares (e.g. Kusano et al., 2012; Bamba et al., 2013) and magnetospheric substorms (e.g. Consolini and Chang, 2001), and in the transfer of mass, momentum, and energy in space and astrophysical plasmas. A key physical process underlying the rearrangement of field line configurations and topology changes is magnetic reconnection, which is known to occur in the solar corona (e.g. Masuda et al., 1994), solar wind (e.g. Gosling et al., 2005), and magnetotail (e.g. Nagai et al., 2013), and at the magnetopause (e.g. Sonnerup et al., 1981). In order to understand in what manner and how efficiently this process converts energy and transfers mass and momentum across a current layer, it is indispensable to reveal the nature of one-dimensional (1-D) discontinuities (rotational or tangential discontinuities and shocks), the formation, location, and interplay of the $\mathrm{X}$ - and O-points in 2-D, and those of magnetic nulls and separators in 3-D (e.g. Cai et al., 2001; Xiao et al., 2006; Wendel and Adrian, 2013), which may be embedded in the current layer in question.

In studies of solar magnetic activities, a number of attempts have been made to reconstruct 3-D force-free magnetic field structures in the corona from nearly instantaneous, remote-sensing (ground-based or space telescope) measurements of the photospheric field (e.g. Wheatland and Leka, 2011; Wiegelmann and Sakurai, 2012; Inoue et al., 2014). In particular, nonlinear force-free field models allow for an 
estimation of the free magnetic energy available, by comparison with reconstructed potential or linear force-free fields, and thus potentially make it possible to forecast where solar flares could be initiated. However, such reconstructions of fully 3-D fields have not been conducted by use of in situ data taken by spacecraft during a short period, while a 3-D magnetohydrostatic equilibrium field in the magnetosphere has been recovered, for example, by modelling the field using Euler potentials and using an average equatorial profile of the plasma pressure based on long-term, in situ measurements as input and empirical fields as boundary conditions (e.g. Zaharia, 2008).

Recently, Sonnerup and Hasegawa (2011) developed a novel data analysis method for the reconstruction of steady, 3-D, magnetohydrostatic structures using plasma and magnetic field data recorded by two closely spaced spacecraft, which is hereafter referred to as the SH11 method. They developed and benchmarked a primitive version of the numerical code for the SH11 method, using an analytical solution of the 3-D magnetohydrostatic equations $(\nabla \cdot \boldsymbol{B}=0$ and $\nabla p=\boldsymbol{j} \times \boldsymbol{B})$. This type of reconstruction in 3-D space, as well as those in 3-D space-time (2-D space and time) developed recently by Sonnerup and Hasegawa (2010), and Hasegawa et al. (2010a, 2014), is a natural extension of a variety of 2-D reconstruction techniques developed to date (see Sonnerup et al., 2006, 2008 and Hasegawa, 2012 for an overview or reviews) that in principle require data from single spacecraft as input. In this paper, we present a first application of the SH11 method to actual observations in space, along with modest improvements of the reconstruction code.

The present paper is organised as follows. In Sect. 2, the basic equations used in the reconstruction and methodology are briefly summarised. In Sect. 3, an overview is given of THEMIS spacecraft observations at the subsolar magnetopause of a flux transfer event (FTE), which is generated through some time-dependent form of magnetopause reconnection. For overviews and models of FTEs, the readers are referred to Scholer (1995), Raeder (2006), and Paschmann et al. (2013). We apply the SH11 3-D reconstruction method as well as the classical Grad-Shafranov (GS) reconstruction technique for 2-D magnetohydrostatic structures (Sonnerup and Guo, 1996; Hau and Sonnerup, 1999; Hasegawa et al., 2004) to the FTE seen by THEMIS and compare the results. In Sect. 4, particle measurements during and around the FTE are analysed in detail to discuss the generation mechanism of the FTE. A brief summary and discussion is presented in Sect. 5.

\section{The method}

We numerically solve the magnetohydrostatic equations using as input the magnetic field and pressure data taken along the paths of two closely separated spacecraft. The assumptions underlying the technique are that the structure to be reconstructed is time-independent and magnetohydrostatic, and moves at a constant velocity relative to the spacecraft. The magnetohydrostatic equations solved in the reconstruction are:

$\nabla \cdot \boldsymbol{B}=0$

and the force balance relation, $\nabla p=\boldsymbol{j} \times \boldsymbol{B}$, which can be written, by use of $\boldsymbol{j}=(\nabla \times \boldsymbol{B}) / \mu_{0}$, in the form

$\nabla P=\frac{(\boldsymbol{B} \cdot \nabla) \boldsymbol{B}}{\mu_{0}}$,

where $P=p+B^{2} /\left(2 \mu_{0}\right)$ is the total (magnetic plus plasma) pressure. Equation (2) assumes that the inertia terms in the MHD equation of motion can be neglected, and expresses the balance between the force from the total pressure gradient and magnetic tension. Equations (1) and (2) constitute four scalar equations for the four unknown physical quantities, $U=\left\{B_{x} ; B_{y} ; B_{z} ; P\right\}$. To understand the reasons why we use the total pressure, rather than plasma pressure, as one of the variables, see Sect. 5 in SH11.

The $x$ axis of the Cartesian reconstruction coordinates is chosen to be parallel to, and halfway between, the paths of the two spacecraft, Sc-A and Sc-B, in the frame co-moving with the structure (Fig. A1). The $z$ axis is defined in such a way that the two spacecraft are contained in the $x-z$ plane. The two spacecraft are separated by $l_{z}$ in the $z$ direction, and are assumed to move in the $+x$ direction at a constant speed, $V_{\text {Sc }}$, relative to the structure. This velocity is usually approximated by the negative deHoffmann-Teller (HT) velocity, determined by the method as described by Khrabrov and Sonnerup (1998), i.e. $\boldsymbol{V}_{\mathrm{Sc}}=-\boldsymbol{V}_{\mathrm{HT}}$. Because of the assumed time independence of the structure, temporal variations seen in time series of the data can now be converted into spatial information along the two spacecraft paths, i.e. spatial variations in the $x$ direction.

We use an equilateral triangular integration grid, as shown in Fig. A1, so that central differences can be used (SH11). Under the assumption of time independency, the $z$ derivatives of $U, \partial U / \partial z$, on the $x$ axis, namely, at the midpoint, $(y, z)=(0,0)$, between the two spacecraft paths, can be evaluated by use of the data taken at the same $x$ locations, i.e. $(\partial U / \partial z)_{i}=\left(U_{B, i}-U_{A, i}\right) / l_{z}$. Here $U_{A, i}$ and $U_{B, i}$ are the values at $(x, y, z)=\left(x_{i}, 0,-l_{z} / 2\right)$ on the path of Sc-A and at $(x, y, z)=\left(x_{i}, 0,+l_{z} / 2\right)$ on the path of Sc-B, respectively. The subscript $i\left(=0,1,2, \ldots, N_{x}=L_{x} / \Delta x\right)$, represents the $i$ th grid point in the $x$ direction, where $L_{x}$ is the $x$ length of the reconstruction domain and $\Delta x$ is the distance between the neighbouring points after interpolation in the $x$ direction of the original data. The $x$ derivatives, $\partial U / \partial x$, on the $x$ axis are calculated by use of a set of the interpolated values at points along the $x$ axis, which are averages, $U_{M, i}=$ $\left(U_{A, i}+U_{B, i}\right) / 2$, of the two spacecraft data at the same $x$ locations, i.e. $(\partial U / \partial x)_{i}=\left(U_{M, i+1}-U_{M, i-1}\right) /(2 \Delta x)$ 
if the lowest-order central difference is used. The four unknown $y$ derivatives $\partial U / \partial y$ are then given by the following equations derived from Eqs. (1) and (2) (SH11),

$$
\frac{\partial U}{\partial y}=\left(\begin{array}{c}
\partial B_{x} / \partial y \\
\partial B_{y} / \partial y \\
\partial B_{z} / \partial y \\
\mu_{0} \partial P / \partial y
\end{array}\right)=
$$

$$
\left(\begin{array}{c}
\left(\mu_{0} / B_{y}\right) \partial P / \partial x-\left(B_{x} / B_{y}\right) \partial B_{x} / \partial x-\left(B_{z} / B_{y}\right) \partial B_{x} / \partial z \\
-\partial B_{x} / \partial x-\partial B_{z} / \partial z \\
\left(\mu_{0} / B_{y}\right) \partial P / \partial z-\left(B_{x} / B_{y}\right) \partial B_{z} / \partial x-\left(B_{z} / B_{y}\right) \partial B_{z} / \partial z \\
B_{x} \partial B_{y} / \partial x+B_{z} \partial B_{y} / \partial z+B_{y}\left(-\partial B_{x} / \partial x-\partial B_{z} / \partial z\right)
\end{array}\right) .
$$

These derivatives can be used to integrate $U$ in the $+y$ direction to obtain the values at $(y, z)=(\Delta y, 0)$ (point 1 in Fig. A1). Here $\Delta y$ is the integration step, which should be adjusted to an optimal value that allows for sufficiently accurate reconstruction over a larger domain and, in Fig. A1, is chosen to satisfy $l_{z}<(2 / \sqrt{3}) \Delta y$. In the present study, $\Delta x$ is set equal to $\Delta y / \sqrt{3}$. In general, $\Delta x$ should be comparable to $\Delta y$ for obtaining the best solution.

The next step is to compute the values at point 2 by use of the extrapolated (or interpolated when $l_{z}>(2 / \sqrt{3}) \Delta y$ ) and integrated values at points $0 \mathrm{~b}$ and 1 , respectively. This can be done by first rotating the coordinate system by $+60^{\circ}$ about the $x$ axis and then using Eq. (3) to integrate $U$ in the rotated $y$ axis direction, i.e. along $+s$ in Fig. A1. This kind of rotation-then-integration process is repeated until the integration reaches the two $z$ boundaries, $z_{\max }$ and $z_{\min }$ (point 9 in Fig. A1). The integration in the plus and minus $y$ direction is then performed by use of Eq. (3) on the basis on the recovered values at $y=\Delta y$ and $y=0$, respectively, and is continued until the two $y$ boundaries are reached. The end results are the reconstructed 3-D distributions of the four quantities $U$, i.e. the 3-D configuration of the magnetic field lines and the pressure distribution, in a rectangular parallelepiped domain.

Since we can use only the lowest-order central difference and two of the four equations (3) have terms divided by $B_{y}$ (and also because of the ill-posed nature of initial value problems), numerical errors develop with increasing number of integration steps, especially in regions where $B_{y}$ reverses sign. The reconstruction is thus possible over only a limited range in the $y$ and $z$ directions, while the $x$ length $L_{x}$ of the reconstruction domain is determined by the path length of the two spacecraft, i.e. $\boldsymbol{V}_{\mathrm{Sc}}$ times the length of the chosen data interval. Because of the numerical errors, the reconstructed total pressure and/or plasma pressure may take on negative values at some grid point(s), after a number of integration steps. Thus, in each integration step, if the computed total pressure value becomes negative, it is reset to zero. If the plasma pressure $p=P-B^{2} /\left(2 \mu_{0}\right)$ computed at the end of all integration steps becomes negative, it is also set to zero. As consequences of these errors and corrections, the reconstructed pressure may not be as strictly preserved along the reconstructed field lines as expected from the magnetohydrostatic force balance equation, and the field may also have nonzero divergence. For details of some newly developed methods to reduce these numerical errors, the readers are referred to Appendix A.

\section{Observations and analysis}

In this section, we present an overview of THEMIS observations on 27 June 2007, 04:50 UT, when an FTE was encountered at the subsolar magnetopause under a southward interplanetary magnetic field (IMF) condition. The FTE is analysed by applying both the 3-D and GS (2-D) reconstruction techniques to the event. Note that both methods assume the magnetohydrostatic force balance. The primary differences are the spatial dimension of the structure reconstructed, and that the 3-D reconstruction requires data from two spacecraft, whereas the Grad-Shafranov reconstruction (GSR) in principle utilises data from a single spacecraft. Hereafter we use lower-case italic letters $(x, y, z)$ for representing the components and positions in GSM, capital italic letters $(X, Y, Z)$ for those in the 3-D reconstruction coordinate system, and capital roman letters (X, Y, Z) for those in the 2-D GSR coordinate system.

\subsection{THEMIS observations of the FTE}

The FTE studied in this paper was encountered by at least three of the five THEMIS probes during the coast phase when they formed a string-of-pearls configuration with separations of order $1 R_{\mathrm{E}}$ (Angelopoulos, 2008). Figure 1 shows data taken by the ESA ion instrument (McFadden et al., 2008) and the fluxgate magnetometer (FGM) (Auster et al., 2008) on board four (THB, THC, THD, and THE) of the five probes during a 14 min interval 04:43-04:57 UT. The probes were moving from the dayside magnetosphere into the subsolar magnetosheath along similar orbits. This is most clearly seen from the THE observations (blue lines in Fig. 1) showing that the ion density, temperature, and the GSM $z$ component of the magnetic field all have typical magnetospheric values at the beginning of the interval, but have magnetosheath values at the end. The IMF observed by THB in the magnetosheath immediately sunward of the magnetopause had a southward component (Fig. 1i), the condition favourable for reconnection to occur at the low latitude magnetopause. THC and THD were separated by about $390 \mathrm{~km}$, THB was on the magnetosheath side of these two probes, and THE was on the magnetospheric side.

Two FTEs were observed during the interval, one at $\sim 04: 46$ UT and another at $\sim$ 04:50 UT. Both events showed typical FTE signatures: a negative to positive perturbation in the $x$ component of the field (Fig. 1g), which is approximately the component normal to the nominal magnetopause 


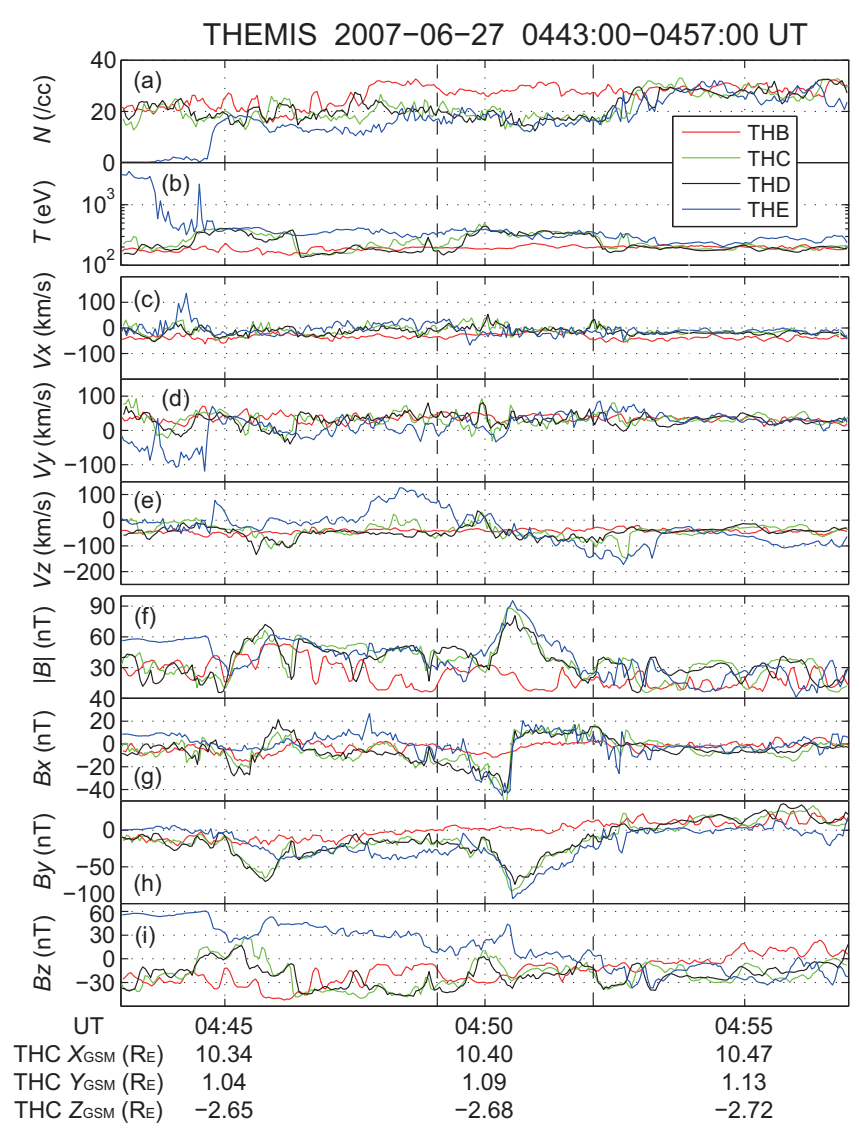

Figure 1. (a-e) Ion and (f-i) magnetic field data in GSM coordinates recorded by the ESA and FGM instruments, respectively, on board four (THB, THC, THD, and THE) of the five THEMIS probes during the interval 27 June 2007, 04:43-04:57 UT. THC and THD data during the interval 04:49:05-04:52:05 UT, sandwiched between the two vertical dashed lines, are used for the 3-D magnetohydrostatic reconstruction.

in the subsolar region, and an increase of the field magnitude (Russell and Elphic, 1978). Here we focus on the second FTE, which is prominent in that the three probes, close to the magnetopause, observed a large negative perturbation of $B_{x}$ (down to about $-40 \mathrm{nT}$ ), and the field intensity at THE exceeded $90 \mathrm{nT}$, much higher than that in the magnetosphere $(\sim 60 \mathrm{nT})$. Note that a rather weak bipolar perturbation in $B_{x}$ was seen by THB in the magnetosheath, almost at the same time as the FTE signatures were seen by the other three probes, and also that around the centre time ( 04:50:30 UT) of the event the core field (seen by THC, THD, and THE) had a strong negative $y$ component even though $B_{y}$ in the magnetosheath as seen by THB was near zero (Fig. 1h).

Interestingly, the second FTE was preceded by a significant northward ion flow at 04:48 UT, followed by a southward flow at 04:52 UT (Fig. 1e). These flows with $\left|v_{z}\right| \geq$ $100 \mathrm{~km} \mathrm{~s}^{-1}$, comparable to the magnetosheath Alfvén speed $\left(\sim 120 \mathrm{~km} \mathrm{~s}^{-1}\right)$, were clearly seen by THE and less clearly by THC, both of which were closer to the Earth than the other two probes (THB and THD), and thus are consistent with reconnection jets observed on the earthward side of a magnetopause of rotational discontinuity-type. Such FTEs sandwiched between oppositely directed reconnection jets have been reported and can be interpreted as being associated with magnetic flux ropes generated through multiple X-line reconnection at the dayside magnetopause (Hasegawa et al., 2010b; Øieroset et al., 2011; Zhang et al., 2012; Zhong et al., 2013; Pu et al., 2013). Their in-depth analysis has demonstrated that the oppositely directed flows are oriented toward the flux rope centre, i.e. X-lines exist on both (northern and southern) sides of the flux rope, and can compress the flux rope from both sides. Such flows are likely to produce the observed larger field magnitude and bipolar $B_{x}$ perturbation at the FTE centre than in FTEs generated by single X-line reconnection.

In the following subsections, we apply both the 3-D and the 2-D (GS) reconstruction methods to the interval between the two vertical dashed lines in Fig. 1. During this interval, the ion flows are not as intense as in the surrounding jet regions where the flow is nearly Alfvénic. Thus the magnetohydrostatic force balance appears to be a reasonable assumption.

\subsection{3-D magnetohydrostatic reconstruction}

The 3-D reconstruction method is applied to a 3 min interval (04:49:05-04:52:05 UT) on 27 June 2007 (between the vertical dashed lines in Fig. 1). For this interval, the HT frame velocity, determined using a set of ion and magnetic field data from both THC and THD, is $\boldsymbol{V}_{\mathrm{HT}}=(-6.3,23.8$, $-56.1) \mathrm{km} \mathrm{s}^{-1}$ in GSM. The field-aligned components of the velocity left over in the HT frame are much smaller than the local Alfvén speed, with the Walén slope of -0.005 (Paschmann and Sonnerup, 2008), which validates the model assumption that inertia effects from the field-aligned flows are negligible. The correlation coefficient between the three components of the magnetic field and velocity in the HT frame is -0.024 , which is equivalent to an average angle between them of $91.4^{\circ}$. This indicates that the velocity components left over in the HT frame were approximately transverse to the magnetic field, so that the structure may have been evolving in a significant way. The correlation coefficient between the three components of the convection electric field and $-\boldsymbol{V}_{\mathrm{HT}} \times \boldsymbol{B}$, converted into the frame in which the average ion velocity for the interval is zero, is 0.240 . This low correlation indicates that the HT frame is not well determined, i.e. the presence of a time-dependent structure. Such possibly non-negligible structural evolution is actually expected from the presence of the oppositely directed jets (Sect. 3.1) that were converging toward the flux rope centre.

Figure 2 shows the 3-D magnetic field and pressure reconstructed, using the data from THC and THD, which were separated by $\sim 390 \mathrm{~km}$ roughly in the GSM $x$ direction. A movie of the 3-D reconstructed field, showing the 3-D field line 

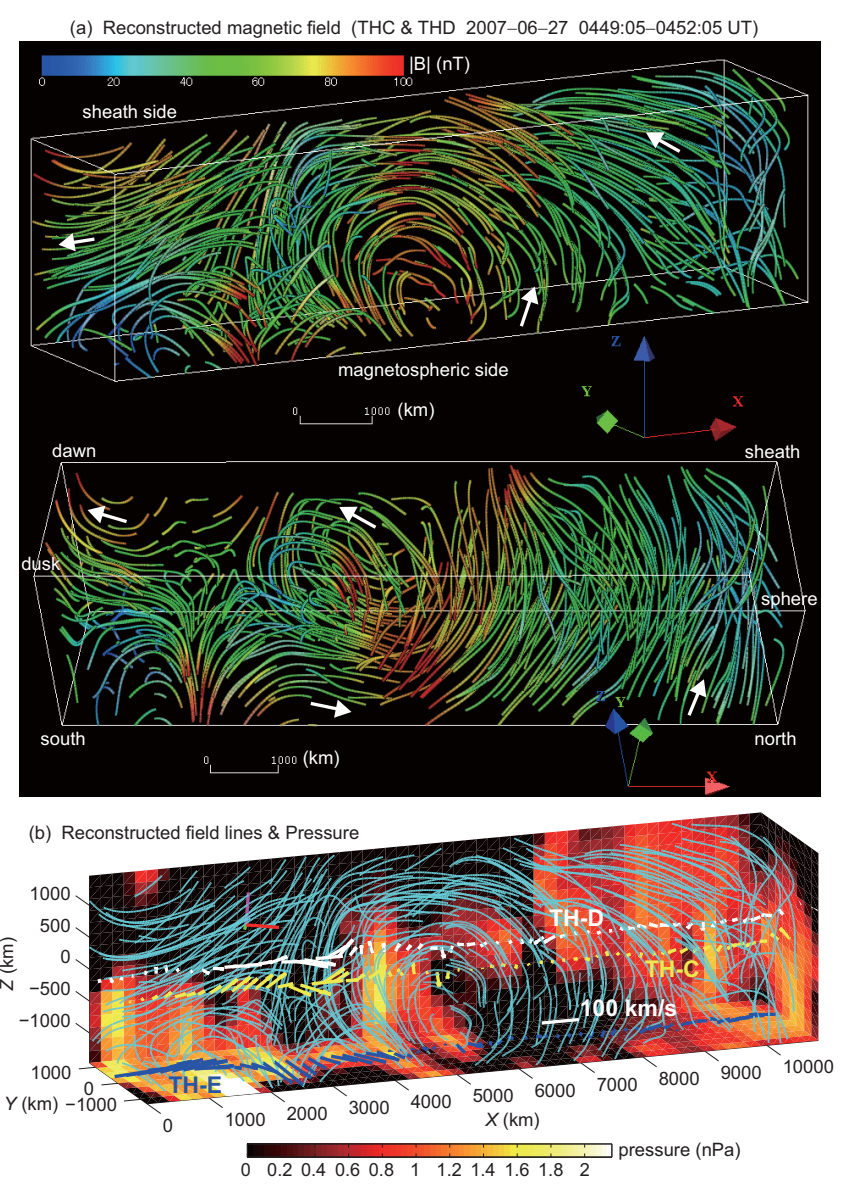

Figure 2. Three-dimensional (3-D) structures of the magnetic field and pressure recovered from the THC and THD data. (a) 3$\mathrm{D}$ representations of the reconstructed field lines, with the field intensity in colour. The top view is roughly from the southdusk, while the bottom view is from the duskside magnetosheath. The three GSM components of the reconstruction axes are: $X=$ $(0.1024,-0.3883,0.9158), Y=(0.0780,-0.9147,-0.3966)$, and $Z=(0.9917,0.1120,-0.0634)$. The white arrows represent the approximate orientation of the magnetic field in the surrounding regions. Please refer to the supplement for a movie of the 3-D reconstructed field, showing the field configuration viewed from various angles. (b) The 3-D field lines (cyan lines) and plasma pressure at the three boundaries of the reconstruction domain in colour. The white, yellow, and blue arrows have roots on the paths of THD, THC, and THE, respectively, and show the ion velocity vectors measured by those probes and transformed into the deHoffmannTeller (HT) frame. The magenta, green, and red bars at the upper left represent the GSM $x, y$, and $z$ axes, respectively.

structure viewed from various angles, is provided in the supplement. The reconstruction axes, $\hat{X}, \hat{Y}$, and $\hat{Z}$, are roughly in the directions of the GSM $z,-y$, and $x$ axes, respectively (see the caption of Fig. 2 for the GSM components of the axes). The separation $l_{z}$ of the two spacecraft along the $Z$ axis is $390.3 \mathrm{~km}$. The integration step $\Delta y$ is set to be $318 \mathrm{~km}$, by maximising the correlation coefficient between the magnetic field components predicted from the reconstruction and those actually measured at points along the path of the THE probe (Fig. 3b). The location of the THE probe relative to the THC location was $(x, y, z)=(-1154,-392,288) \mathrm{km}$ in GSM, and $(X, Y, Z)=(298,154,-1206) \mathrm{km}$ in the reconstruction coordinates. Bear in mind that the reconstructed fields near the boundary surfaces may not be reliable, because the numerical errors accumulate with increasing number of integration steps, i.e. with distance from the $X$ axis. The recovered 3-D field lines in Fig. 2a unambiguously demonstrate that a structure of magnetic flux rope-type was encountered by THC and THD. The intensity of the recovered field has a maximum $(\sim 100 \mathrm{nT})$ near the centre of the reconstruction box, consistent with the presence of a flux rope. The cross section of the flux rope had a diameter of $\sim 3000 \mathrm{~km}$.

In the top panel of Fig. 2a, the field structure may look approximately 2-D. However, the recovered field lines appear to intersect each other at many points in this projection plane (viewed from a certain angle), suggesting that this FTE had a pronounced 3-D structure. A comparison between the results from the SH11 method and from the GS reconstruction, which will be presented in Sect. 3.3, indeed demonstrates that the observed structure is better described by a 3-D, rather than 2-D, model. The velocity vectors transformed into the HT frame, denoted by the arrows in Fig. 2b, are generally oriented toward the centre of the flux rope, although the speed is relatively low on the northern side (in the right part of the figure) of the centre. The flow directions are compatible with the oppositely directed jets observed on the southern and northern sides of the flux rope, i.e. with the presence of a reconnection X-line on both sides, as discussed in Sect. 3.1. These converging flows would have compressed the flux rope and would have resulted in the intense core field at its centre (Fig. 1f and h).

Figure 3 shows a comparison between the three GSM components of the magnetic field predicted along the THE path from the 3-D reconstruction and those actually measured by the THE probe. Since the observed field variations are generally similar at the three probes, it may not be surprising that the variation predicted at the THE location agrees well with the observed variation. We point out, however, that as clearly shown in the right part of Fig. 3 a (middle panel), the magnitude of $B_{y}$ observed at THE is larger than at THC and THD, and that the polarity of $B_{z}$ seen by THE was different from that at THC and THD (bottom panel) since THE was on the magnetospheric side of the FTE centre. It is striking that these differences are well recovered by the 3-D reconstruction.

Figure $3 \mathrm{~b}$ shows the correlation between the GSM components of the magnetic field predicted and actually measured by THE. The Bootstrap method is used for estimating the confidence interval of the correlation coefficient (e.g. Efron and Tibshirani, 1986, 1994; Kawano and Higuchi, 1995). The resulting confidence interval corresponding to \pm 1 sigma is from 0.8642 to 0.9020 . The correlation coeffi- 

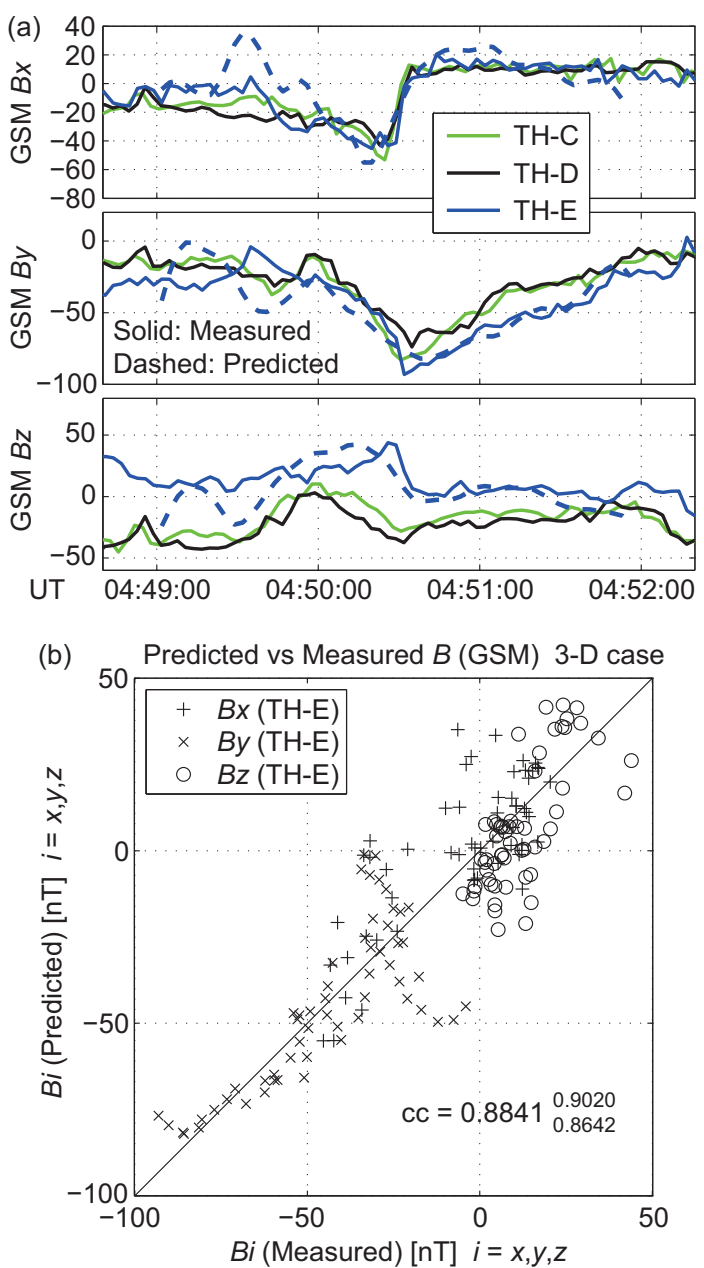

Figure 3. (a) Time series of three GSM components of the magnetic field actually measured (solid) and predicted (dashed) along the THE path (blue), along with the THC (green) and THD (black) data used for the 3-D reconstruction. (b) Correlation between the three GSM components of the predicted and measured fields at THE, but excluding the data points closest to the $X$ boundaries of the reconstruction domain. The subscript and superscript of the correlation coefficient represent the upper and lower values of the confidence interval corresponding to \pm 1 sigma.

cient $(0.8841)$ is not particularly high and is lower than those seen for high-latitude FTEs reported and successfully reconstructed with GS reconstruction by Sonnerup et al. (2004) and Hasegawa et al. (2006). This lower correlation is likely due to a significant evolution of the structure during the time interval in question. A measure of structural evolution over the interval is $M_{\mathrm{A}}\left(T_{\mathrm{int}} / \tau_{\mathrm{A}}\right)=0.89$, one order of magnitude higher than for the events studied by Hasegawa et al. (2014). Here, $M_{\mathrm{A}}$ is the average plasma speed $\left(29.7 \mathrm{~km} \mathrm{~s}^{-1}\right)$ in the HT frame divided by the magnetosheath Alfvén speed $\left(V_{\mathrm{A}}=120 \mathrm{~km} \mathrm{~s}^{-1}\right), T_{\text {int }}$ is the analysis interval $(3 \mathrm{~min})$, and $\tau_{\mathrm{A}}=L / V_{\mathrm{A}}$ is the Alfvén transit time where the characteristic length scale of the structure $L=6000 \mathrm{~km}$ ). The long event duration, which is partially responsible for the higher value of $M_{\mathrm{A}}\left(T_{\mathrm{int}} / \tau_{\mathrm{A}}\right)$, results from a relatively low HT frame speed, namely, slow motion of the FTE flux rope near the subsolar magnetopause. Nonetheless, we emphasise that the correlation coefficient $\mathrm{CC}=0.8841_{0.8642}^{0.9020}$ is significantly higher than that $\left(0.8007_{0.7753}^{0.8259}\right)$ from the GS reconstruction of the present FTE, because the confidence interval for the 3-D case is above that for the 2-D case (see Sect. 3.3 and Fig. 7 for details).

\subsection{2-D Grad-Shafranov reconstruction}

Here we apply the single-spacecraft version of the GS reconstruction technique (Sonnerup and Guo, 1996; Hau and Sonnerup, 1999; Hasegawa et al., 2004) to THC observations of the FTE during the same 3 min interval (04:49:0504:52:05 UT) as for the 3-D reconstruction, and compare the 2-D reconstruction results with those from the 3-D reconstruction presented in Sect. 3.2. THC was closer to THE than THD (THC was at $1252 \mathrm{~km}$ from THE, and THD was at $1631 \mathrm{~km} /$ from THE). Thus, the GS reconstruction using THC data would and does better predict the field variations at THE than that using THD data (Fig. 7). For details of the methodology, the readers are referred to Hau and Sonnerup (1999) and Sonnerup et al. (2006).

The HT velocity determined from the THC data alone is $V_{\mathrm{HT}}=(-4.7,13.7,-56.8) \mathrm{km} \mathrm{s}^{-1}$ in GSM. The Walén slope is -0.028 , and the correlation coefficient between the field and velocity components in the HT frame is -0.124 , equivalent to the average angle between the field and velocity of $97.1^{\circ}$. These values are similar to those resulting from the combined THC and THD data used in the 3-D reconstruction. The invariant $(Z)$ axis, along which gradient is assumed to be negligible $(\partial / \partial \mathrm{Z} \approx 0)$, is determined by the method used by Hasegawa et al. (2004), i.e. through maximisation of the correlation coefficient between the three field components predicted at points along the THE path and those actually measured. Note that only THE data are used in the calculation of the correlation coefficient in order to make the comparison with the 3-D result (Fig. 3b) easier. Remember that the GSR X axis is defined to be parallel to the projection of the THC path (in the HT frame) onto the plane perpendicular to the $\mathrm{Z}$ axis, i.e. is antiparallel to the projection of $\boldsymbol{V}_{\mathrm{HT}}$, and that the $\mathrm{Y}$ axis completes the right-handed orthogonal system.

Figure 4 shows the transverse pressure, $P_{t}=p+$ $B_{Z}^{2} /\left(2 \mu_{0}\right)$, and the axial component of the magnetic field $B_{Z}$ as functions of the partial vector potential $A$, for the determined orientation of the invariant axis. In principle, these two quantities should be preserved along the field lines in 2-D GS equilibria (e.g. Sonnerup et al., 2006), and this property has been used in the method, developed by Hu and Sonnerup (2002), for estimating the axial (Z) orientation of magnetic flux ropes on the basis of single-spacecraft measurements. However, both panels in Fig. 4 indicate that the data 

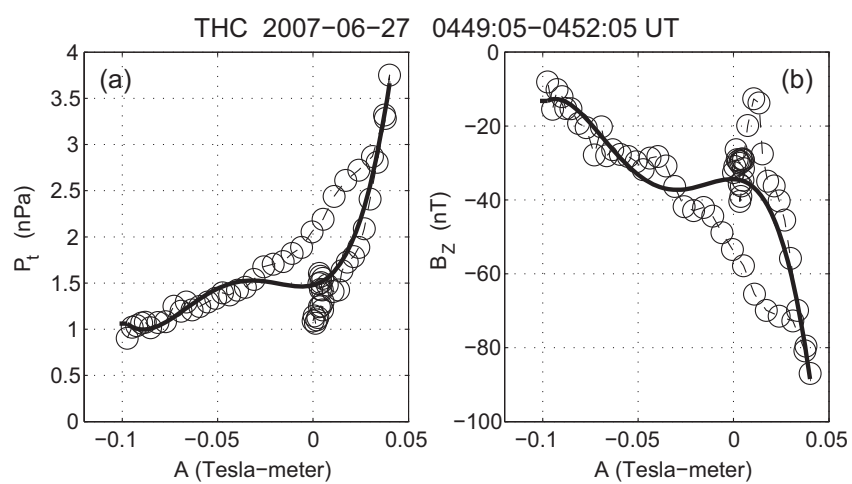

Figure 4. Scatter plots of (a) the transverse pressure, $P_{t}=p+$ $B_{Z}^{2} /\left(2 \mu_{0}\right)$, versus partial vector potential $A$ and (b) of the axial magnetic field component $B_{Z}$ versus $A$, for the invariant ( $\mathrm{Z}$ ) axis orientation estimated by maximising the correlation coefficient (Fig. 6b) between the field components measured and predicted, from the Grad-Shafranov reconstruction (GSR) using the THC data (Fig. 5), along the THE path. The circles show the measurements and the thick black curves are polynomial fits to the measurements.

points acquired by THC during the FTE interval are not represented by a single curve, but show a double-branch feature. We also estimated the axis by the $\mathrm{Hu}$ and Sonnerup method in such a way that the measured data points in the $P_{t}$ versus $A$ space (as shown in Fig. 4a) fall on a single curve as best as possible. The resultant axis, $\mathrm{Z}=(0.1114,0.9228,0.3688)$, has a substantial angle $\left(16.1^{\circ}\right)$ with respect to the correlation coefficient-based axis. These features imply that the FTE in question cannot be well represented by a 2-D model, but has a significant 3-D structure.

Figure 5 shows the 2-D magnetic field and pressure maps reconstructed by the GSR method. The three THEMIS probes traversed the structure at $56.8 \mathrm{~km} \mathrm{~s}^{-1}$ along the $\mathrm{X}$ axis, from left to right in the map. The 2-D results suggest that the probes encountered a magnetic flux rope with a diameter of $\sim 3000 \mathrm{~km}$, essentially consistent with the 3$D$ reconstruction results. However, the transverse (in-plane) components of the recovered magnetic field are not parallel to those actually measured at points along the THE path, especially for the earlier half of the interval corresponding to the southern side of the FTE centre; in the left part of the map (Fig. 5a), the black curves have substantial angles with respect to the white arrows on the THE path.

Figure 6 shows a time series and scatter plot of the three GSM components of the magnetic field predicted from the 2D map at points along the THE path and those actually measured. The panels indicate that the reconstructed fields do not agree well with the measured fields. As seen in Fig. 7, the correlation coefficients in the 2-D case, $\mathrm{CC}=0.8007_{0.7753}^{0.8259}$ for the THC-based GSR and CC $=0.7136_{0.6717}^{0.7566}$ for the THD-based GSR, are indeed significantly lower than that $\left(0.8841_{0.8642}^{0.9020}\right)$ in the 3 -D case. The quantity $1-\mathrm{CC}^{2}$, which is a measure of the deviations, is 0.36 for GSR using the
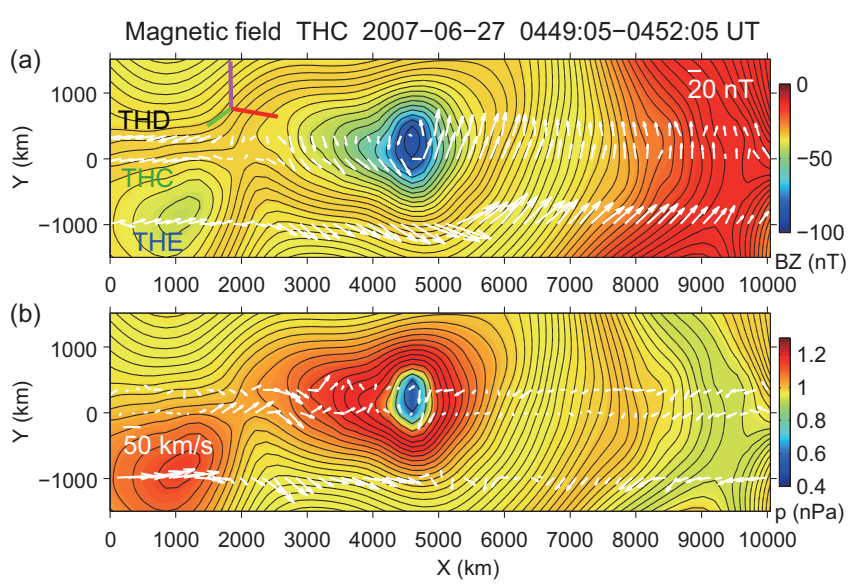

Figure 5. Two-dimensional (2-D) magnetic field and pressure recovered from the GSR method using the THC data for the same interval as for the 3-D reconstruction. Black lines show the reconstructed transverse field lines, with (a) the axial field component $B_{Z}$ and (b) pressure in colour. White arrows show the projections onto the GSR X-Y plane of the actually measured magnetic fields in panel (a) and ion velocities left over in the HT frame in panel (b). The GSR axes are in GSM: $\mathrm{X}=(-0.0131,-0.4544,0.8907), \mathrm{Y}=$ $(0.9295,-0.3339,-0.1567)$, and $\mathrm{Z}=(0.3686,0.8258,0.4268)$. The magenta, green, and red bars in panel (a) are the GSR X-Y projections of the GSM $x, y$, and $z$ axes, respectively.

THC data and 0.22 for the 3-D reconstruction. These comparisons demonstrate that the FTE encountered by THEMIS had a substantial 3-D magnetic structure, and that the SH11 method can provide the 3-D field of acceptable quality for an actually observed structure.

\section{Magnetic topology of the FTE}

In this section, we investigate magnetic field-aligned and anti-field-aligned fluxes of electrons, as well as of ions observed in the FTE and surrounding regions. Energy-versustime spectrograms of the ion fluxes can be used to identify signatures of ion acceleration or heating, possibly associated with magnetopause reconnection, while field-aligned streaming electrons can be used as tracers of the topology of the field lines. Figure 8 shows the spectrograms of the differential energy fluxes $\left(\mathrm{eV} \mathrm{cm}^{-2} \mathrm{~s}^{-1} \mathrm{ster}^{-1} \mathrm{eV}^{-1}\right)$ in the spacecraft frame based on the THD and THE observations, along with the magnetic field data from the four probes presented in Fig. 1. The THC spectrograms look similar to the ones for THD (Fig. 8e-h) and thus are not shown, while THB (on the magnetosheath side) was too far from the FTE core to be of help in revealing the topological properties. Note that THD traversed the central but somewhat magnetosheath-side part of the FTE, whereas THE was on the magnetospheric side of THD and THC (Fig. 2).

For the 14 min interval shown in Fig. 8, THD observed either the magnetosheath or the magnetopause boundary layer 
(a)
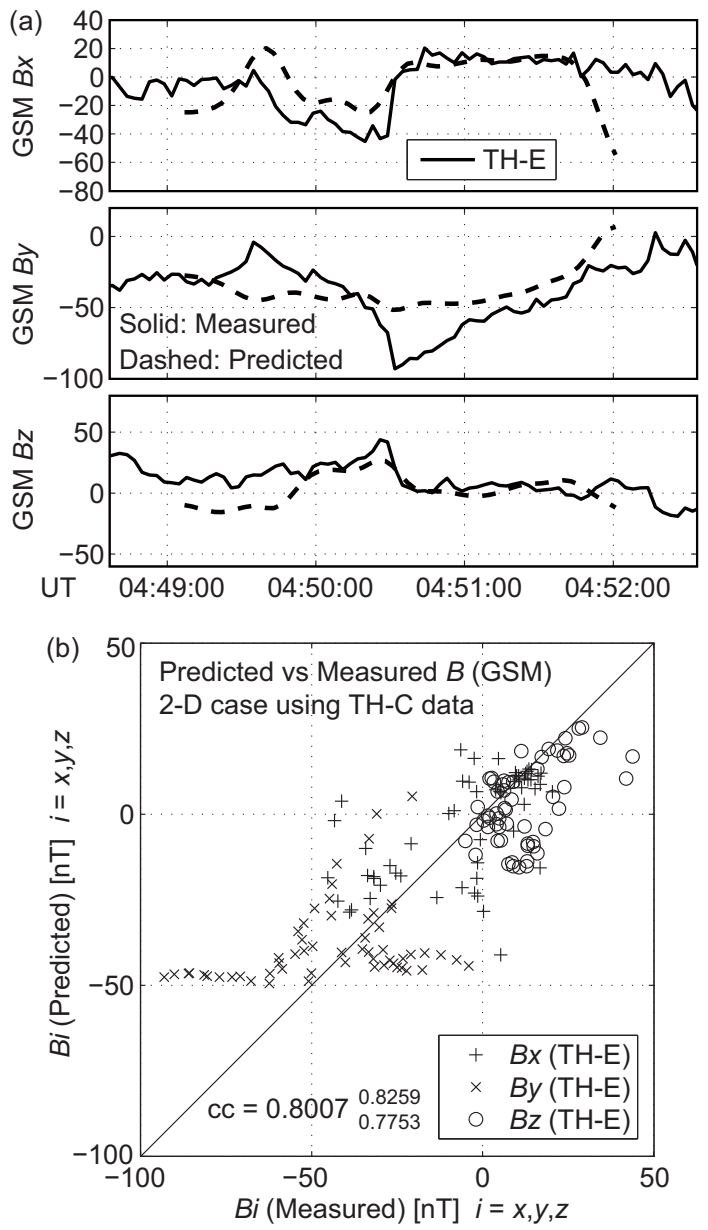

Figure 6. The same as Fig. 3 but for the 2-D Grad-Shafranov reconstruction using the THC data. The field variations along only the THE path are shown.

in which FTEs were embedded, while THE observed either the typical outer magnetospheric region or the boundary layers including the magnetosheath boundary layer (MSBL) (e.g. Fuselier, 1995). The magnetosheath, encountered by THD, e.g. after 04:53 UT, was dominated by ions with energies less than a few $\mathrm{keV}$ and electrons with energies less than a few hundreds eV (Fig. 8e-h). As discussed in Sect. 3, two FTEs were encountered at around 04:46 and 04:50 UT, when both the ion and electron spectrograms were characterised by the coexistence of the hot ( $>$ a few $\mathrm{keV}$ ) magnetospheric population and heated or accelerated magnetosheath population. During a part of the second FTE interval to which the reconstruction methods were applied (Sect. 3), the fieldaligned and anti-field-aligned electron fluxes were approximately balanced at all energies (Fig. 9), suggesting that the field lines are closed, i.e. anchored to the Earth at both ends (e.g. Øieroset et al., 2008; Pu et al., 2013). However, at $\sim 04: 50: 30$ UT near the centre of the FTE flux rope, the anti-field-aligned flux was significantly higher than the field-

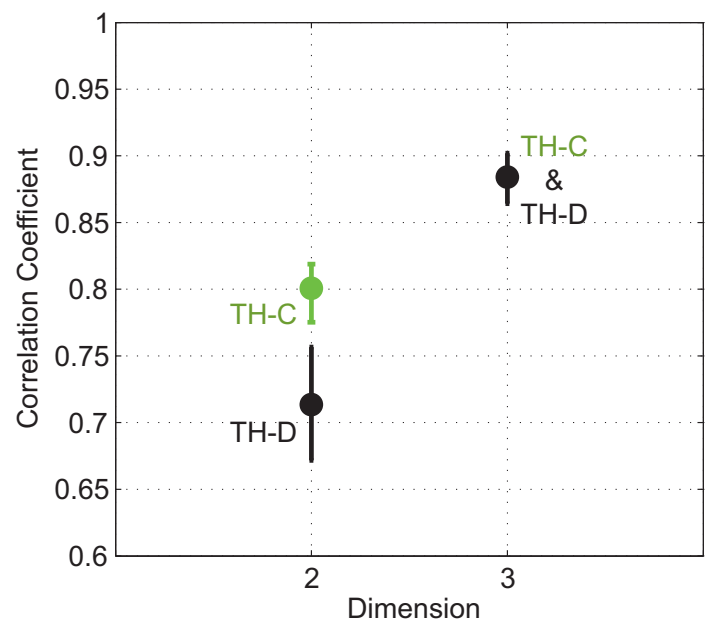

Figure 7. The correlation coefficients between the three GSM components of the predicted and measured fields at THE, and their confidence intervals corresponding to \pm 1 sigma, for the 2-D and 3-D methods. The two correlation coefficients in the 2-D case are from the GS reconstruction applied individually to the THC and THD data.

aligned flux at energies of more than a few $\mathrm{keV}$. Such electron pitch-angle anisotropy within FTEs generated through multiple X-line reconnection has been reported by Øieroset et al. (2011) and Pu et al. (2013). It indicated that the field lines near the FTE centre were open, connected to the northern ionosphere at one end and extending to interplanetary space at the other end, in this particular case.

Heated magnetosheath electrons were seen by THD throughout the FTE in both the field-aligned and anti-fieldaligned fluxes (Fig. 8g and h). We note that these bidirectional heated electrons were observed on the magnetosheath side of the FTE centre where $B_{z}<0$, and thus were not due to THD crossing into the magnetosphere or its boundary layer. This feature can be taken as a signature of magnetopause reconnection on the field lines traversed by the spacecraft (e.g. Onsager et al., 2001). For this particular event, it indicated that reconnection occurred on both the northern and southern sides of the FTE, i.e. the observed FTE was formed by multiple X-line reconnection (e.g. Hasegawa et al., 2010b). These electron pitch-angle distribution signatures demonstrate that FTEs generated by multiple X-line reconnection can consist of field lines of various topologies, as shown by $\mathrm{Pu}$ et al. (2013). On the other hand, at $\sim 04: 48: 35$ and $\sim$ 04:52:50 UT in the MSBL immediately on the magnetosheath side of the FTE, the field-aligned (southward streaming) electrons showed no heated magnetosheath population while the anti-field-aligned (northward streaming) electrons showed the heated population. This indicated that the MSBL field lines observed there crossed the magnetopause on the southern side only of the FTE (Hasegawa et al., 2010b). 


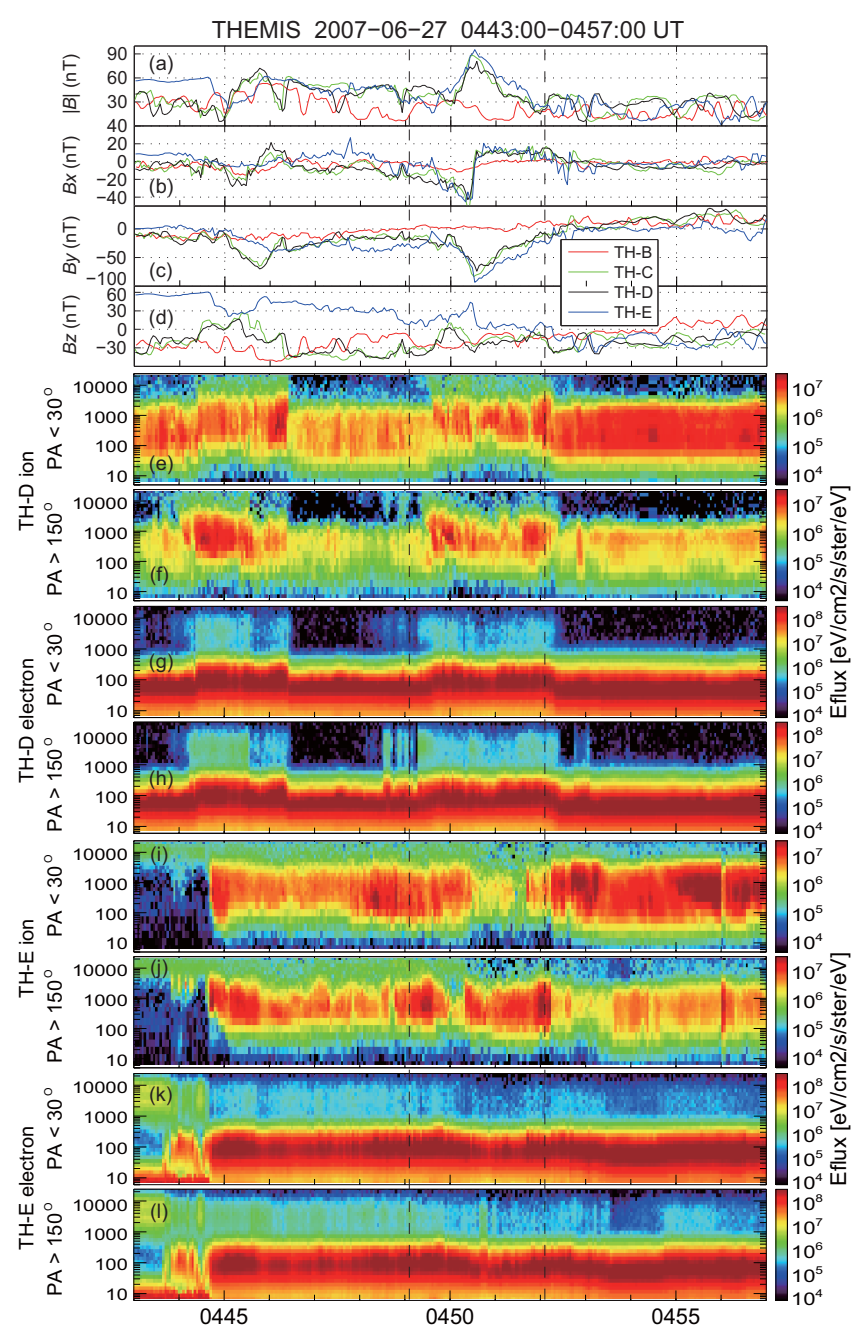

Figure 8. (a-d) Magnetic field measurements by the four THEMIS probes, and (e-l) energy-versus-time spectrograms of ions and electrons detected by the THD and THE probes. PA stands for the "pitch-angle" in the spacecraft frame of particles in question, and thus the upper and lower panels in each set of the two ion or electron spectrograms show the differential energy fluxes of field-aligned and anti-field-aligned particles, respectively. The reconstruction interval is sandwiched between the two vertical dashed lines.

THE was initially in the magnetosphere, dominated by ions and electrons of more than $1 \mathrm{keV}$, but after $\sim 04: 44 \mathrm{UT}$ was in the boundary layers, either earthward $\left(B_{z}>0\right)$ or sunward $\left(B_{z}<0\right)$ of the magnetopause (Fig. 8i-1). Both boundary layers are characterised by the coexistence of magnetospheric and magnetosheath populations. However, the fluxes of magnetospheric electrons and energies of magnetosheath ions were both somewhat lower in the MSBL, seen by THE after $\sim 04: 53$ UT when the field became southward, than in the boundary layer on the magnetospheric side. The magnetospheric electrons observed near the FTE centre ( 04:50:45 UT) had a pitch-angle anisotropy similar to that seen by THD at $\sim 04: 50: 30 \mathrm{UT}$; the field lines in the FTE
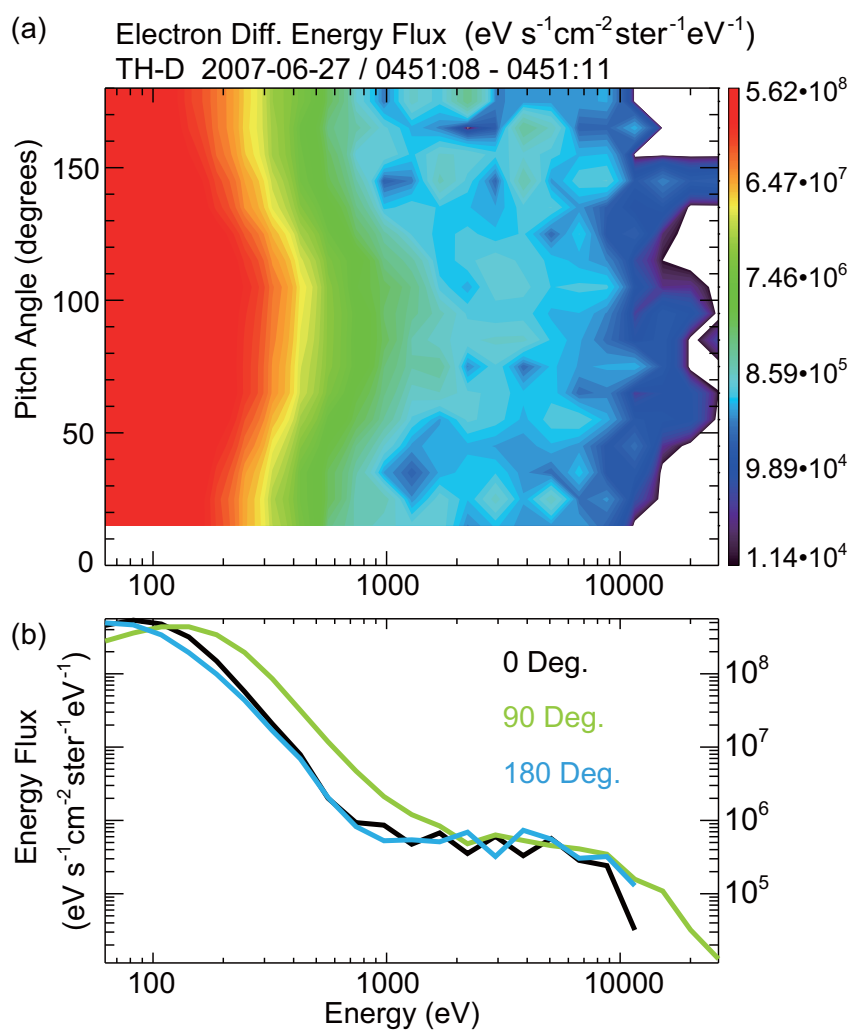

Figure 9. (a) Electron pitch-angle versus energy spectrogram and (b) energy distributions at the pitch angles 0,90 , and $180^{\circ}$, obtained in the FTE by THD at 04:51:08-04:51:11 UT. The field-aligned and anti-field-aligned fluxes are roughly balanced especially at higher energies, indicating that the field lines in the FTE are partly closed.

core were open. Interestingly, the field-aligned and anti-fieldaligned electron fluxes were roughly balanced throughout the MSBL interval of THE (Fig. 8k, 1), suggesting that the MSBL field lines were closed. Possible explanations for this feature are given and discussed in Sect. 5. In summary, the THD and THE particle signatures are all consistent with the view that the FTE resulted from multiple $\mathrm{X}$-line reconnection at the low-latitude magnetopause and consisted of a mixture of closed field lines and open ones with one end connected to the northern ionosphere and the other end extending to interplanetary space.

\section{Summary and discussion}

We have presented the first results of the data analysis technique, developed by Sonnerup and Hasegawa (2011), for reconstructing steady 3-D magnetohydrostatic magnetic field and plasma structures from dual-spacecraft observations. The method was applied to data taken at $~ 04: 50$ UT on 27 June 2007 by the THC and THD probes of the THEMIS spacecraft during a flux transfer event (FTE). The event occurred at/around the subsolar magnetopause, when the IMF 
had a southward component and the two probes were separated by $\sim 390 \mathrm{~km}$. The results can be summarised as follows:

1. Structure: the 3-D reconstruction results show that a magnetic flux rope with a diameter of $\sim 3000 \mathrm{~km}$ was embedded in the FTE. Comparison between the 3-D reconstruction and 2-D GS reconstruction results indicates that the flux rope had a substantial 3-D structure, not well described by 2-D models. The flux rope was elongated roughly in the dawn-dusk direction, with a left-handed chirality, i.e. the core field had a significant dawnward component.

2. Time dependence: the perpendicular (to the field) components of the velocity remaining in the HT frame were fairly large, suggesting that the flux rope was still evolving. Since the FTE was sandwiched between two oppositely directed reconnection jets and the flow was generally oriented toward the centre of the flux rope, it is likely that the flux rope was being compressed by the jets from both sides. We were not able to successfully reconstruct the flux rope by the methods, developed by Sonnerup and Hasegawa (2010) and Hasegawa et al. (2010a, 2014), that can recover slow time evolution of 2-D magnetohydrostatic structures. This may indicate that the 3-D and/or time-dependent effects were too strong to be accommodated by the 2-D time evolution methods.

3. Magnetic topology: the pitch-angle distribution of hot magnetospheric electrons shows that the FTE consisted of open and closed field lines. A major part of the flux rope was on closed field lines, consistent with its generation by multiple $\mathrm{X}$-line reconnection; a significant fraction of open field lines produced at one X-line was later closed by reconnection at another X-line. However, the field lines around the centre of the flux rope were open, with one end anchored to the northern ionosphere. This suggests that during an initial phase of the generation, the reconnected flux tube of the observed FTE was on the northern side of an X-line, i.e. reconnection occurred first on the southern, rather than northern, side of the FTE.

4. Generation mechanism: all the observed features, such as the observed locations of the oppositely directed ion jets relative to the FTE and electron pitch-angle distributions, indicate that multiple $\mathrm{X}$-line reconnection was involved in the FTE formation. The FTE flux rope was generally moving southward, probably pushed by the southward reconnection jet located on the northern side of the FTE. A likely sequence of magnetopause reconnection, first on the southern side of the FTE and later on the northern side under the condition when the magnetic dipole axis is tilted sunward in the northern hemisphere (see also Hasegawa et al., 2010b), is consistent with the FTE generation mechanism proposed by Raeder (2006) in which multiple X-line reconnection occurs sequentially under a substantial tilt of the geomagnetic dipole axis.

5. Implication for the 3-D method: the results demonstrate that our 3-D reconstruction method provides a 3-D magnetic field of acceptable quality when the THC and THD probes, which acquired input data for the reconstruction, are separated by $\sim 390 \mathrm{~km}$, about $10 \%$ of the size of the FTE, in the direction transverse to the probe paths in the chosen structure-rest (HT) frame. This suggests that the method may be used for a not-too-limited range of inter-spacecraft distance (of the order of 1 to $10 \%$ of the characteristic scale length). Remember, however, that our earlier benchmark test (Sonnerup and Hasegawa, 2011) shows that an optimal separation is only a few per cent of the characteristic scale length.

6. Implication for the 2-D methods: for substantial 3-D structures, 2-D methods (e.g. Hu and Sonnerup, 2002; Hasegawa et al., 2004; Sonnerup and Hasegawa, 2005) can fail to estimate the orientation of the invariant axis, along which the spatial gradient is assumed to be negligible. A significant difference among the axial orientations estimated by various 2-D methods may indicate the existence of a 3-D structure. Such cases should be interpreted with care. On the other hand, the 2-D GS method may help to roughly estimate the size and shape of a 3-D structure, as demonstrated by Hasegawa et al. (2007).

Although the present version of 3-D reconstruction using two-spacecraft data is restricted to applications to steady magnetohydrostatic structures, it is in principle possible to extend the method to those applicable to steady fully MHD or Hall MHD structures. As discussed by Sonnerup and Hasegawa (2011), a more general case would be reconstruction of not only 3-D features but also time dependent effects, which requires the use of data from at least three closely spaced spacecraft. A difficulty associated with these more sophisticated reconstructions is that they require such well calibrated data, for all MHD or Hall MHD parameters (including ion velocity and electric field) and from a larger number of spacecraft, so that precise values of spatial and/or temporal gradients can be estimated. The present method requires magnetic field and pressure data of sufficient quality from only two spacecraft (or the field data alone under low $\beta$ conditions). Another disadvantage would be that the equations used in the more sophisticated versions could have more singularities, leading to larger numerical errors. These are the issues that should be addressed in future studies.

It is worth noting that a fraction of the low-latitude boundary layer (LLBL) seen by THD and THE was characterised 
by approximately balanced fluxes of field-aligned and antifield-aligned electrons (Figs. 8 and 9) and thus appeared to be on closed field lines, even under a southward IMF condition. The formation of such a closed LLBL requires magnetopause reconnection at more than one site, as proposed by Nishida (1989) and Song and Russell (1992), unless diffusion is responsible for its formation. Our observations indeed show that multiple $\mathrm{X}$-line reconnection was involved in the generation of the observed FTE. Since the oppositely directed plasma jets emanating from different reconnection sites may collide, the associated open flux tubes may interact with each other and be entangled in a complex way (Fig. 4 in Nishida, 1989). Louarn et al. (2004) indeed reported a signature of such entangled or interlinked reconnected flux tubes. A possible scenario that can explain our observations is that reconnection of these open flux tubes resulted in the formation of the closed flux tube containing solar wind plasma and of the IMF-type flux tube (Fig. 2 in Nishida, 1989), thus creating the closed portion of the LLBL present in and around the FTE. For another possible way to create the closed field lines in the flux rope, see Fig. 2 in Pu et al. (2013). We point out that in an FTE reported by Øieroset et al. (2011), there was no evidence of reconnection in the central part of the flux rope flanked by two active $\mathrm{X}$-lines where two reconnection jets and thus reconnected flux tubes from the two Xlines were colliding (Øieroset et al., 2014). Thus, the question of whether the Nishida mechanism works in reality remains open.

Another possible consequence of multiple X-line reconnection at the low-latitude magnetopause under southward IMF is less efficiency in the transfer of solar wind energy to the magnetotail than with the transfer resulting from single X-line reconnection (Hasegawa et al., 2010b; Hasegawa, 2012). Because an $X$-line may exist ahead of a reconnection jet emanating from another $\mathrm{X}$-line, magnetic flux tubes reconnected and eroded on the dayside may be entangled or interlinked (Nishida, 1989) and may not be smoothly transported from dayside to nightside. Thus, if multiple X-line reconnection occurs more frequently under larger geomagnetic dipole tilt, as suggested by Raeder (2006), lowering the energy transfer rate, it may be that the total amount of solar wind energy deposited to the magnetotail is smaller during summer and winter, possibly contributing to seasonal variations of geomagnetic activities. Although it may be difficult to observationally confirm such a possible relationship among the occurrence of multiple X-line reconnection, dipole tilt, and solar wind energy transfer rate, global magnetospheric simulations may help to reveal or refute the connection.

Interesting questions that can be addressed with the 3D reconstruction method are whether an FTE, as hypothesised originally by Russell and Elphic (1978), exists and to what extent such FTEs contribute to magnetic flux transport toward the tail. In the Russell and Elphic model, FTEs are generated when magnetopause reconnection occurs in- termittently and locally on a short segment of single X-line, and are characterised by an elbow-shaped (namely fully 3D) flux tube connecting the magnetosheath and magnetosphere. Such localised and transient reconnection is indeed shown to be able to produce a bipolar variation in the magnetic field component normal to the nominal current layer (e.g. Semenov et al., 1994; Shirataka et al., 2006). However, unambiguous identification of the Russell-Elphic type FTE would need to demonstrate that the local orientation of the flux tube axis at around the elbow has a nonzero angle with respect to the magnetopause surface. It requires accurate estimation of the magnetopause normal as well as of the orientation of the flux tube or rope. The latter would be possible by careful analysis of the magnetic gradient tensor that can be computed from the reconstructed data at any point in the 3 -D reconstruction domain. We also need some reasonable way for determining the boundary of the flux tube or rope and magnetic flux content from the 3-D field data (and other additional information) in order to be able to assess the role of FTEs. These subjects will be pursued in a future study.

The 3-D magnetic field recovered by methods of the type presented here can in principle be used to calculate the spatial gradient of the field (Shi et al., 2005) and current density $\left(\boldsymbol{j}=(\nabla \times \boldsymbol{B}) / \mu_{0}\right)$ at any point in the reconstruction domain, and also to identify magnetic nulls, separators, and quasi-separatrix layers (Priest and Demoulin, 1995; Cai et al., 2001; Xiao et al., 2006; Wendel and Adrian, 2013; Komar et al., 2013), key ingredients in 3-D reconnection that could potentially exist in the domain. Recent particle simulations show that 3-D dynamics can play an important role in the magnetic dissipation in collisionless reconnection (Che et al., 2011; Fujimoto and Sydora, 2012) and the evolution of reconnecting current sheets (Daughton et al., 2011, 2014; Nakamura et al., 2013). Our expectation is that the 3-D reconstruction technique, combined with other multispacecraft methods and data from NASA's forthcoming Magnetospheric MultiScale (MMS) mission (Burch and Drake, 2009; Moore et al., 2013), will facilitate our understanding of the 3-D aspects of magnetic reconnection. 


\section{Appendix A: Minimising numerical errors}

In this section, we describe two methods to reduce numerical errors associated with the spatial integration used in our 3-D reconstruction. Major sources of the errors are (i) that two of the four equations in (3), used in the integration, have terms divided by $B_{y}$ and thus have a singularity when $B_{y}$ vanishes, and (ii) that only the lowest-order central difference can be used to estimate the $z$ derivatives, since we suppose that data from only two spacecraft are available. The methods are validated by using an analytical solution of the 3D magnetohydrostatic equations describing an axially symmetric spheromak field geometry. This field appears 3-D in Cartesian coordinates, and was used in benchmark tests of a primitive numerical code of the 3-D reconstruction (Sonnerup and Hasegawa, 2011).

In one of the methods, we make a correction of the $y$ derivatives when $\partial U / \partial y$ estimated by Eq. (3) exceeds a certain threshold value. As an example, let us consider a step in which the values at point $\alpha$ in the $y-z$ plane in Fig. A1 are computed. Suppose $U$ to be the quantities normalised to their typical value, $(\partial U / \partial y)_{j}$ to be the $y$ derivatives estimated by using Eq. (3) based on the values at points 1 and 2, and $(\partial U / \partial y)_{j-1 / 2}$ to be those estimated using the values at point $0 \mathrm{~b}$ and at the midpoint between points 1 and 2, i.e.

$\left(\frac{\partial U}{\partial y}\right)_{j-1 / 2}=\frac{\left(U_{1}+U_{2}\right) / 2-U_{0 b}}{\Delta y}$.

Here $U_{1}, U_{2}$, and $U_{0 b}$ are the values at points 1,2 , and $0 \mathrm{~b}$, respectively, and simple linear interpolation is used to obtain the values at the midpoint. Substantial difference between $(\partial U / \partial y)_{j}$ and $(\partial U / \partial y)_{j-1 / 2}$ suggests that members of $(\partial U / \partial y)_{j}$ are not of acceptable accuracy likely because of the appearance of the singularities or the use of the low-order scheme. Thus, we replace $(\partial U / \partial y)_{j}$ by $(\partial U / \partial y)_{j-1 / 2}$ if the absolute value of the difference $\left|(\partial U / \partial y)_{j}-(\partial U / \partial y)_{j-1 / 2}\right|$ exceeds a certain threshold value (set at 0.5 in the present study). Note that this correction is possible in all steps, except for the first step to compute the values at point 1 . For example, the computation and evaluation of the values at point 2 , conducted in the second step, are made using the values at points $1,0 \mathrm{~b}$, and $0 \mathrm{a}$.

The other method, implemented after the first one, concerns the solenoidal property of the magnetic field, $\nabla \cdot \boldsymbol{B}=0$, and tries to reduce nonzero divergence of the reconstructed field. Although $\nabla \cdot \boldsymbol{B}=0$ is explicitly used in Eq. (3) to compute $\partial B_{y} / \partial y$, nonzero divergence may appear after the correction described above and smoothing in the $x$ direction of $\partial U / \partial y$ are performed in each integration step. Such smoothing is needed to minimise the effects of unrealistically large absolute values (even after the above correction) and/or an oscillatory behaviour in the $x$ direction of $\partial U / \partial y$ numerically estimated (Sonnerup and Hasegawa, 2011). The value

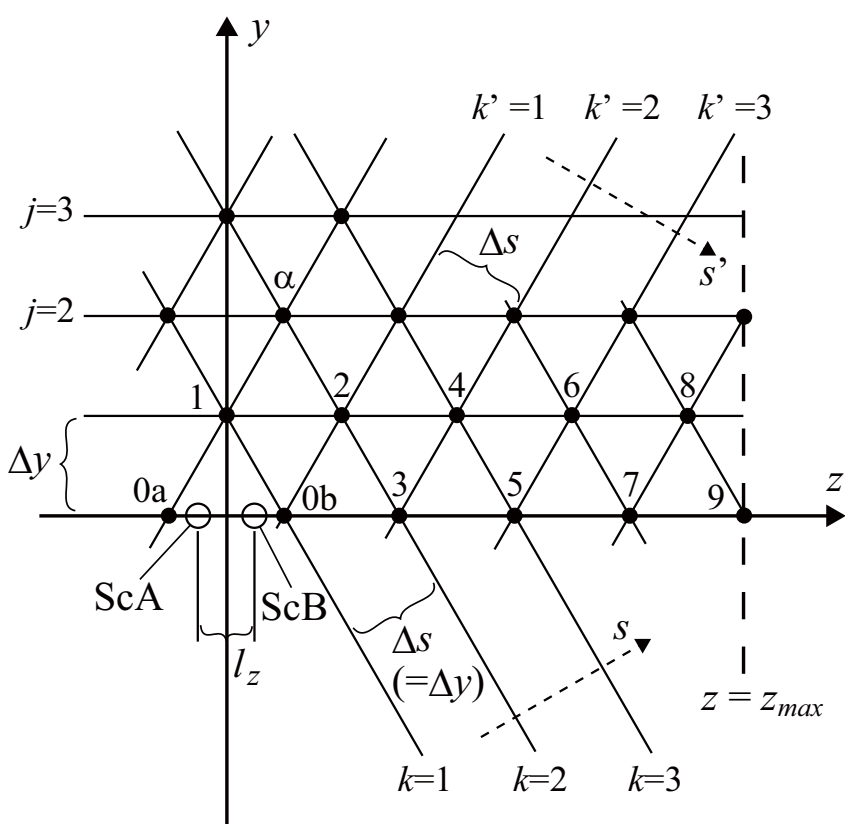

Figure A1. Triangular central-difference integration grid used in the reconstruction (modified from Fig. A1 of SH11). The $x$ axis is halfway between the paths of Sc-A and Sc-B, and points into the figure plane. Numbers on the grid points indicate the order in which data at them are calculated. See text for details about the methods used to reduce numerical errors in estimating the gradients.

of $\nabla \cdot \boldsymbol{B}$ after the smoothing can be written as:

$\nabla \cdot \boldsymbol{B}_{j, \text { old }}=\left(\frac{\partial B_{x}}{\partial x}\right)_{j}+\left(\frac{\partial B_{y}}{\partial y}\right)_{j, \text { old }}+\left(\frac{\partial B_{z}}{\partial z}\right)_{j}=\beta_{j}$,

where $\beta_{j}$ may be nonzero. We nudge the numerically estimated $\partial B_{y} / \partial y$ toward satisfying $\nabla \cdot \boldsymbol{B}=0$ in the following way:

$\left(\frac{\partial B_{y}}{\partial y}\right)_{j, \text { new }}=\left(\frac{\partial B_{y}}{\partial y}\right)_{j, \text { old }}-C \beta_{j}$,

where $C$ is a nudging factor smaller than unity (set at 0.5 in the present study), and the subscripts "old" and "new" represent the values before and after the nudging correction, respectively. The corrected value $\left(\partial B_{y} / \partial y\right)_{j, \text { new }}$ is used in the estimation of $\partial P / \partial y$ as well as $\partial B_{y} / \partial y$ (note that the terms in the parenthesis on the right-hand side of the fourth of Eq. (3) are in total equal to $\partial B_{y} / \partial y$ ).

In Fig. A2, we compare numerical solutions of the poloidal fields and plasma pressure from three versions of the 3-D integration, one with no corrections of $\partial U / \partial y$ and $\nabla \cdot \boldsymbol{B}$ (panel b), one with only $\partial U / \partial y$ correction (panel c), and one used in the application with both $\partial U / \partial y$ and $\nabla \cdot \boldsymbol{B}$ corrections. With no corrections, the recovered field lines deviate appreciably from those of the exact solution near the boundaries of the reconstruction domain (Fig. A2b). The pressure is 
(a) Exact solution of Pressure \& field lines

(b) Recovered Pressure \& field lines

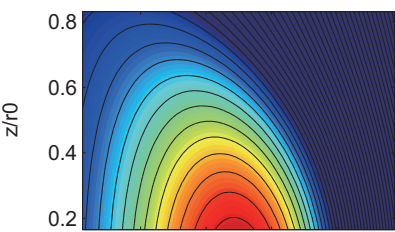

No $\operatorname{grad}(U) \& \operatorname{div}(B)$ corrections

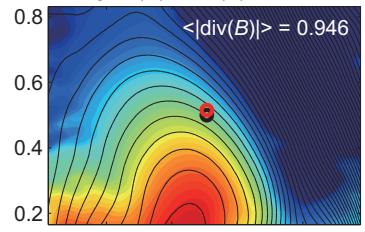

(c) Recovered Pressure \& field lines

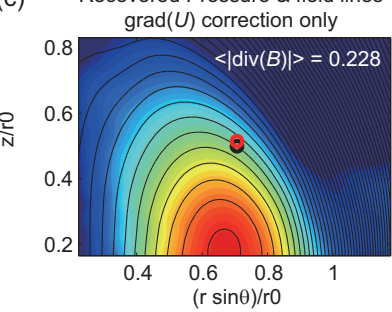

(d) Recovered Pressure \& field lines Both $\operatorname{grad}(U) \& \operatorname{div}(B)$ corrections

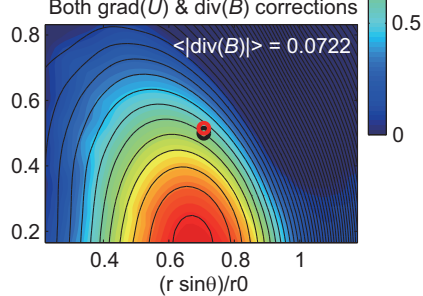

Figure A2. Comparison between (a) exact solution and benchmark reconstructions with (b) no corrections, (c) $\nabla U$ correction only, and (d) both $\nabla U$ and $\nabla \cdot \boldsymbol{B}$ corrections (see Appendix for details). The format is the same as in the right panels in Fig. 6a of SH11; poloidal field lines are shown in a meridional plane at $\varphi=\pi / 4$, with plasma pressure in colour. Small circles indicate the locations at which the Sc-A (black) and Sc-B (red) paths cross the plane. The number at the upper-right of each panel shows the mean absolute value of $\nabla \cdot \boldsymbol{B}$, as a measure of the quality of the numerical integration.

also not preserved along the field lines, contrary to expectation from the magnetohydrostatic force balance $\nabla p=\boldsymbol{j} \times \boldsymbol{B}$. The $\partial U / \partial y$ correction makes the reconstructed fields in good agreement with the exact ones and makes the pressure roughly constant along the field lines, but the divergence of the field is still substantial (Fig. A2c). With both corrections, the mean absolute value of the divergence is reduced to 0.0722 (Fig. A2d), which is only $\sim 32 \%$ of that for the version with the $\partial U / \partial y$ correction only. The comparison demonstrates that the two methods described above are helpful in significantly reducing the numerical errors. 


\section{The Supplement related to this article is available online at doi:10.5194/angeo-33-169-2015-supplement.}

Acknowledgements. The authors thank the THEMIS team for successful management and operation of the mission and for allowing our use of the data from the ESA and FGM instruments. Work by $\mathrm{H}$. Hasegawa at JAXA was supported by JSPS Grant-in-Aid for Scientific Research KAKENHI grant no. 24740337. Work at Dartmouth College was partially supported by NASA grant NNX14AC38G.

Topical editor E. Roussos thanks A. Isavnin and one anonymous referee for their help in evaluating this paper.

\section{References}

Angelopoulos, V.: The THEMIS mission, Space Sci. Rev., 141, 534, 2008.

Auster, H.-U., Glassmeier, K.-H., Magnes, W., Aydogar, O., Baumjohann, W., Constantinescu, D., Fischer, D., Fornacon, K. H., Georgescu, E., Harvey, P., Hillenmaier, O., Kroth, R., Ludlam, M., Narita, Y., Nakamura, R., Okrafka, K., Plaschke, F., Richter, I., Schwarzl, H., Stoll, B., Valavanoglou, A., and Wiedemann, M.: The THEMIS fluxgate magnetometer, Space Sci. Rev., 141, 235-264, 2008.

Bamba, Y., Kusano, K., Yamamoto, T. T., and Okamoto, T. J.: Study on the triggering process of solar flares based on HINODE/SOT observations, Astrophys. J., 778, 48, doi:10.1088/0004-637X/778/1/48, 2013.

Burch, J. L., and Drake, J. F.: Reconnecting magnetic fields, American Scientist, 97, 392-399, 2009.

Cai, D.-S., Li, Y., Ichikawai, T., Xiao, C., and Nishikawa, K.: Visualization and criticality of magnetotail field topology in a threedimensional particle simulation, Earth Planet. Space, 53, 10111019, 2001

Che, H., Drake, J. F., and Swisdak, M.: A current filamentation mechanism for breaking magnetic field lines during reconnection, Nature, 474, 184-187, doi:10.1038/nature10091, 2011.

Consolini, G. and Chang, T. S.: Magnetic field topology and criticality in geotail dynamics: Relevance to substorm phenomena, Space Sci. Rev., 95, 309-321, doi:10.1023/A:1005252807049, 2001.

Daughton, W., Roytershteyn, V., Karimabadi, H., Yin, L., Albright, B. J., Bergen, B., and Bowers, K. J.: Role of electron physics in the development of turbulent magnetic reconnection in collisionless plasmas, Nature Phys., 7, 539-542, doi:10.1038/nphys1965, 2011.

Daughton, W., Nakamura, T. K. M., Karimabadi, H., Roytershteyn, V., and Loring, B.: Computing the reconnection rate in turbulent kinetic layers by using electron mixing to identify topology, Phys. Plasmas, 21, 052307, doi:10.1063/1.4875730, 2014.

Efron, B. and Tibshirani, R.: Bootstrap methods for standard errors, confidence intervals, and other measures of statistical accuracy, Statist. Sci., 1, 54-75, 1986.

Efron, B. and Tibshirani, R. J.: An introduction to the bootstrap, Vol. 57, CRC Press, Boca Raton, FL., 1994.
Fujimoto, K. and Sydora, R.: Plasmoid-induced turbulence in collisionless magnetic reconnection, Phys. Rev. Lett., 109, 265004, doi:10.1103/PhysRevLett.109.265004, 2012.

Fuselier, S. A.: Kinetic aspects of reconnection at the magnetopause, in Physics of the magnetopause, Geophys. Monogr. Ser. vol. 90, edited by: Song, P., Sonnerup, B. U. Ö., and Thomsen, M. F., 181-187, AGU, Washington, DC, 1995.

Gosling, J. T., Skoug, R. M., McComas, D. J., and Smith, C. W.: Direct evidence for magnetic reconnection in the solar wind near 1 AU, J. Geophys. Res., 110, A01107, doi:10.1029/2004JA010809, 2005.

Hasegawa, H.: Structure and dynamics of the magnetopause and its boundary layers, Monogr. Environ. Earth Planets, 1, 71-119, doi:10.5047/meep.2012.00102.0071, 2012.

Hasegawa, H., Sonnerup, B. U. Ö., Dunlop, M. W., Balogh, A., Haaland, S. E., Klecker, B., Paschmann, G., Dandouras, I., Lavraud, B., and Rème, H.: Reconstruction of two-dimensional magnetopause structures from Cluster observations: verification of method, Ann. Geophys., 22, 1251-1266, doi:10.5194/angeo-221251-2004, 2004.

Hasegawa, H., Sonnerup, B. U. Ö., Owen, C. J., Klecker, B., Paschmann, G., Balogh, A., and Rème, H.: The structure of flux transfer events recovered from Cluster data, Ann. Geophys., 24, 603-618, doi:10.5194/angeo-24-603-2006, 2006.

Hasegawa, H., Nakamura, R., Fujimoto, M., Sergeev, V. A., Lucek, E. A., Rème, H., and Khotyaintsev, Y.: Reconstruction of a bipolar magnetic signature in an earthward jet in the tail: Flux rope or 3D guide-field reconnection?, J. Geophys. Res., 112, A11206, doi:10.1029/2007JA012492, 2007.

Hasegawa, H., Sonnerup, B. U. Ö., and Nakamura, T. K. M.: Recovery of time evolution of Grad-Shafranov equilibria from single-spacecraft data: Benchmarking and application to a flux transfer event, J. Geophys. Res., 115, A11219, doi:10.1029/2010JA015679, 2010a.

Hasegawa, H., Wang, J., Dunlop, M. W., Pu, Z. Y., Zhang, Q.H., Lavraud, B., Taylor, M. G. G. T., Constantinescu, O. D., Berchem, J., Angelopoulos, V., McFadden, J. P., Frey, H. U., Panov, E. V., Volwerk, M., and Bogdanova, Y. V.: Evidence for a flux transfer event generated by multiple X-line reconnection at the magnetopause, Geophys. Res. Lett., 37, L16101, doi:10.1029/2010GL044219, 2010b.

Hasegawa, H., Sonnerup, B. U. Ö., Hu, Q., and Nakamura, T. K. M.: Reconstruction of an evolving magnetic flux rope in the solar wind: Decomposing spatial and temporal variations from single-spacecraft data, J. Geophys. Res. Space Physics, 119, doi:10.1002/2013JA019180, 2014.

Hau, L.-N. and Sonnerup, B. U. Ö.: Two-dimensional coherent structures in the magnetopause: Recovery of static equilibria from single-spacecraft data, J. Geophys. Res. Space Phys., 104, 6899-6917, 1999.

Hu, Q. and Sonnerup, B. U. Ö.: Reconstruction of magnetic clouds in the solar wind: Orientation and configuration, J. Geophys. Res., 107, 1142, doi:10.1029/2001JA000293, 2002.

Inoue, S., Magara, T., Pandey, V. S., Shiota, D., Kusano, K., Choe, G. S., and Kim, K. S.: Nonlinear force-free extrapolation of the coronal magnetic field based on the magnetohydrodynamic relaxation method, Astrophys. J., 780, 101, doi:10.1088/0004637X/780/1/101, 2014. 
Kawano, H. and Higuchi, T.: The bootstrap method in space physics: Error estimation for minimum variance analysis, Geophys. Res. Lett., 22, 307-310, doi:10.1029/94GL02969, 1995.

Khrabrov, A. V. and Sonnerup, B. U. Ö.: DeHoffmann-Teller Analysis, in Analysis Methods for Multi-Spacecraft Data, edited by: Paschmann, G. and Daly, P. W., Chap. 9, Int. Space Sci. Inst., Bern, Switzerland, and Eur. Space Agency, Paris, France, 221248, 1998.

Komar, C. M., Cassak, P. A., Dorelli, J. C., Glocer, A., and Kuznetsova, M. M.: Tracing magnetic separators and their dependence on IMF clock angle in global magnetospheric simulations, J. Geophys. Res. Space Phys., 118, 4998-5007, doi:10.1002/jgra.50479, 2013.

Kusano, K., Bamba, Y., Yamamoto, T. T., Iida, Y., Toriumi, S., and Asai, A.: Magnetic field structures triggering solar flares and coronal mass ejections, Astrophys. J., 760, 31, doi:10.1088/0004-637X/760/1/31, 2012.

Louarn, P., Fedorov, A., Budnik, E., Fruit, G., Sauvaud, J. A., Harvey, C. C., Dandouras, I., Rème, H., Dunlop, M. W., and Balogh, A.: Cluster observations of complex 3D magnetic structures at the magnetopause, Geophys. Res. Lett., 31, L19805, doi:10.1029/2004GL020625, 2004.

Masuda, S., Kosugi, T., Hara, H., Tsuneta, S., and Ogawara, Y.: A loop-top hard X-ray source in a compact solar flare as evidence for magnetic reconnection, Nature, 371, 495-497, doi:10.1038/371495a0, 1994.

McFadden, J. P., Carlson, C. W., Larson, D., Ludlam, M., Abiad, R., Elliott, B., Turin, P., Marckwordt, M., and Angelopoulos, V.: The THEMIS ESA plasma instrument and in-flight calibration, Space Sci. Rev., 141, 277-302, 2008.

Moore, T. E., Burch, J. L., Daughton, W. S., Fuselier, S. A., Hasegawa, H., Petrinec, S. M., and Pu, Z.: Multiscale studies of the three-dimensional dayside X-line, J. Atmos. Sol.-Terr. Phys., 99, 32-40, doi:10.1016/j.jastp.2012.10.004, 2013.

Nagai, T., Shinohara, I., Zenitani, S., Nakamura, R., Nakamura, T. K. M., Fujimoto, M., Saito, Y., and Mukai, T.: Three-dimensional structure of magnetic reconnection in the magnetotail from Geotail observations, J. Geophys. Res. Space Physics, 118, 16671678, doi:10.1002/jgra.50247, 2013.

Nakamura, T. K. M., Daughton, W., Karimabadi, H., and Eriksson, S.: Three-dimensional dynamics of vortex-induced reconnection and comparison with THEMIS observations, J. Geophys. Res. Space Physics, 118, 5742-5757, doi:10.1002/jgra.50547, 2013.

Nishida, A.: Can random reconnection on the magnetopause produce the low latitude boundary layer?, Geophys. Res. Lett., 16, 227-230, 1989.

Øieroset, M., Phan, T. D., Angelopoulos, V., Eastwood, J. P., McFadden, J., Larson, D., Carlson, C. W., Glassmeier, K.-H., Fujimoto, M., and Raeder, J.: THEMIS multi-spacecraft observations of magnetosheath plasma penetration deep into the dayside low-latitude magnetosphere for northward and strong By IMF, Geophys. Res. Lett., 35, L17S11, doi:10.1029/2008GL033661, 2008.

Øieroset, M., Phan, T. D., Eastwood, J. P., Fujimoto, M., Daughton, W., Shay, M. A., Angelopoulos, V., Mozer, F. S., McFadden, J. P., Larson, D. E., and Glassmeier, K.-H.: Direct evidence for a threedimensional magnetic flux rope flanked by two active magnetic reconnection X lines at Earth's magnetopause, Phys. Rev. Lett., 107, 165007, doi:10.1103/PhysRevLett.107.165007, 2011.
Øieroset, M., Sundkvist, D., Chaston, C. C., Phan, T. D., Mozer, F. S., McFadden, J. P., Angelopoulos, V., Andersson, L., and Eastwood, J. P., Observations of plasma waves in the colliding jet region of a magnetic flux rope flanked by two active $\mathrm{X}$ lines at the subsolar magnetopause, J. Geophys. Res. Space Physics, 119, 6256-6272, doi:10.1002/2014JA020124, 2014.

Onsager, T. G., Scudder, J. D., Lockwood, M., and Russell, C. T.: Reconnection at the high-latitude magnetopause during northward interplanetary magnetic field conditions, J. Geophys. Res. Space Phys., 106, 25467-25488, doi:10.1029/2000JA000444, 2001.

Paschmann, G. and Sonnerup, B. U. Ö.: Proper frame determination and Walén test, in Multi-spacecraft analysis methods revisited, edited by: Paschmann, G. and Daly, P. W., ISSI SR-008, 65-74, ESA Publications Division, 2008.

Paschmann, G., Øieroset, M., and Phan, T.: In-situ observations of reconnection in space, Space Sci. Rev., 47, 309-341, doi:10.1007/978-1-4899-7413-6_12, 2013.

Priest, E. R. and Demoulin, P.: Three-dimensional magnetic reconnection without null points: 1 . Basic theory of magnetic flipping, J. Geophys. Res., 100, 23443-23463, doi:10.1029/95JA02740, 1995.

Pu, Z. Y., Raeder, J., Zhong, J., Bogdanova, Y. V., Dunlop, M., Xiao, C. J., Wang, X. G., and Fazakerley, A.: Magnetic topologies of an in vivo FTE observed by Double Star/TC-1 at Earth's magnetopause, Geophys. Res. Lett., 40, 3502-3506, doi:10.1002/grl.50714, 2013.

Raeder, J.: Flux Transfer Events: 1. generation mechanism for strong southward IMF, Ann. Geophys., 24, 381-392, doi:10.5194/angeo-24-381-2006, 2006.

Russell, C. T. and Elphic, R. C.: Initial ISEE magnetometer results: magnetopause observations, Space Sci. Rev., 22, 681-715, 1978

Scholer, M.: Models of flux transfer events, in Physics of the magnetopause, Geophys. Monogr. Ser., Vol. 90, edited by: Song, P., Sonnerup, B. U. O., and Thomsen, M. F., 235-245, AGU, Washington, DC, 1995.

Semenov, V. S., Farrugia, C. J., Biernat, H. K., Levedeva, V. V., and Rijnbeek, R. P.: Reconnection-associated surface waves at the magnetopause, Geophys. Res. Lett., 21, 2437-2440, 1994.

Shi, Q. Q., Shen, C., Pu, Z. Y., Dunlop, M. W., Zong, Q.-G., Zhang, H., Xiao, C. J., Liu, Z. X., and Balogh, A.: Dimensional analysis of observed structures using multipoint magnetic field measurements: Application to Cluster, Geophys. Res. Lett., 32, L12105, doi:10.1029/2005GL022454, 2005.

Shirataka, N., Fujimoto, M., Hasegawa, H., and TanDokoro, R.: Reproducing the bipolar magnetic signature at the jet leading edge by three-dimensional reconnection with nonzero guide field, J. Geophys. Res., 111, A07201, doi:10.1029/2005JA011521, 2006.

Song, P. and Russell, C. T.: Model of the formation of the lowlatitude boundary layer for strongly northward interplanetary magnetic field, J. Geophys. Res., 97, 1411-1420, 1992.

Sonnerup, B. U. Ö. and Guo, M.: Magnetopause transects, Geophys. Res. Lett., 23, 3679-3682, 1996.

Sonnerup, B. U. Ö. and Hasegawa, H.: Orientation and motion of two-dimensional structures in a space plasma, J. Geophys. Res., 110, A06208, doi:10.1029/2004JA010853, 2005.

Sonnerup, B. U. Ö. and Hasegawa, H.: On slowly evolving Grad-Shafranov equilibria, J. Geophys. Res., 115, A11218, doi:10.1029/2010JA015678, 2010. 
Sonnerup, B. U. Ö. and Hasegawa, H.: Reconstruction of steady, three-dimensional, magnetohydrostatic field and plasma structures in space: Theory and benchmarking, J. Geophys. Res. Space Phys., 116, A09230, doi:10.1029/2011JA016675, 2011.

Sonnerup, B. U. Ö., Paschmann, G., Papamastorakis, I., Sckopke, N., Haerendel, G., Bame, S. J., Asbridge, J. R., Gosling, J. T., and Russell, C. T.: Evidence for magnetic field reconnection at the Earth's magnetopause, J. Geophys. Res., 86, 10049-10067, 1981.

Sonnerup, B. U. Ö., Hasegawa, H., and Paschmann, G.: Anatomy of a flux transfer event seen by Cluster, Geophys. Res. Lett., 31, L11803, doi:10.1029/2004GL020134, 2004.

Sonnerup, B. U. Ö., Hasegawa, H., Teh, W.-L., and Hau, L.-N.: Grad-Shafranov reconstruction: An overview, J. Geophys. Res., 111, A09204, doi:10.1029/2006JA011717, 2006.

Sonnerup, B. U. Ö., Teh, W.-L., and Hasegawa, H.: Grad-Shafranov and MHD reconstructions, in Multi-spacecraft analysis methods revisited, edited by: Paschmann, G. and Daly, P. W., ISSI SR008, 81-90, ESA Publications Division, 2008.

Wendel, D. E. and Adrian, M. L.: Current structure and nonideal behavior at magnetic null points in the turbulent magnetosheath, J. Geophys. Res. Space Physics, 118, 1571-1588, doi:10.1002/jgra.50234, 2013.

Wheatland, M. S. and Leka, K. D.: Achieving self-consistent nonlinear force-free modeling of solar active regions, Astrophys. J., 728, 112. doi:10.1088/0004-637X/728/2/112, 2011.
Wiegelmann, T. and Sakurai, T.: Solar force-free magnetic fields, Living Rev. Solar Phys., 9, 5, doi:10.12942/lrsp-2012-5, 2012.

Xiao, C. J., Wang, X. G., Pu, Z. Y., Zhao, H., Wang, J. X., Ma, Z. W., Fu, S. Y., Kivelson, M. G., Liu, Z. X., Zong, Q. G., Glassmeier, K. H., Balogh, A., Korth, A., Reme, H., and Escoubet, C. P.: In situ evidence for the structure of the magnetic null in a $3 \mathrm{D}$ reconnection event in the Earth's magnetotail, Nature Phys., 2, 478-483, doi:10.1038/nphys342, 2006.

Zaharia, S.: Improved Euler potential method for three-dimensional magnetospheric equilibrium, J. Geophys. Res., 113, A08221, doi:10.1029/2008JA013325, 2008.

Zhang, H., Kivelson, M. G., Angelopoulos, V., Khurana, K. K., Pu, Z. Y., Walker, R. J., McPherron, R. L., Hsu, T.S., Zong, Q. G., and Phan, T.: Generation and properties of in vivo flux transfer events, J. Geophys. Res., 117, A05224, doi:10.1029/2011JA017166, 2012.

Zhong, J., Pu, Z. Y., Dunlop, M. W., Bogdanova, Y. V., Wang, X. G., Xiao, C. J., Guo, R. L., Hasegawa, H., Raeder, J., Zhou, X. Z., Angelopoulos, V., Zong, Q. G., Fu, S. Y., Xie, L., Taylor, M. G. G. T., Shen, C., Berchem, J., Zhang, Q. H., Volwerk, M., and Eastwood, J. P.:: Three-dimensional magnetic flux rope structure formed by multiple sequential X-line reconnection at the magnetopause, J. Geophys. Res. Space Physics, 118, 1904-1911, doi:10.1002/jgra.50281, 2013. 